\title{
Remarks on infinitely many solutions for a class of Schrödinger equations with sign-changing potential
}

Rong Cheng ${ }^{1 *}$ and Yijia Wu'

\section{"Correspondence:}

mathchr@163.com

${ }^{1}$ School of Mathematics and

Statistics, Nanjing University of

Information Science and

Technology, Nanjing, P.R. China

\section{Springer}

\begin{abstract}
In this paper, we study the existence of infinitely many nontrivial solutions for the following semilinear Schrödinger equation:

$$
\left\{\begin{array}{l}
-\Delta u+V(x) u=f(x, u), \quad x \in \mathbb{R}^{N}, \\
u \in H^{1}\left(\mathbb{R}^{N}\right),
\end{array}\right.
$$

where the potential $V$ is continuous and is allowed to be sign-changing. By using a variant fountain theorem, we obtain the existence of infinitely many high energy solutions under the condition that the nonlinearity $f(x, u)$ is of super-linear growth at infinity. The super-quadratic growth condition imposed on $F(x, u)=\int_{0}^{u} f(x, t) d t$ is weaker than the Ambrosetti-Rabinowitz type condition and the similar conditions employed in the references.
\end{abstract}

MSC: $34 C 25 ; 37 J 45$

Keywords: Variational method; Schrödinger equation; Multiple solutions; Fountain theorem

\section{Introduction and main result}

In the present paper, we are concerned with the existence of infinitely many nontrivial solutions for the following semilinear Schrödinger equation:

$$
\left\{\begin{array}{l}
-\Delta u+V(x) u=f(x, u), \quad x \in \mathbb{R}^{N} \\
u \in H^{1}\left(\mathbb{R}^{N}\right) .
\end{array}\right.
$$

Due to the strong background of (1.1) in many fields, such as physics, optics, etc., it has attracted a great deal of interest in the past several decades. Here we point out that a variety of types of existent results on solutions for (1.1) have been considered by different assumptions on potential $V(x)$ and nonlinearity $f(x, u)$ by applying variational methods. For the case of $V(x)$ and $f(x)$ being nonperiodic, many authors have studied the existence of nontrivial solutions for problem (1.1) with $f(x)$ being superlinear in [1-3], sublinear

(c) The Author(s) 2020. This article is licensed under a Creative Commons Attribution 4.0 International License, which permits use, sharing, adaptation, distribution and reproduction in any medium or format, as long as you give appropriate credit to the original author(s) and the source, provide a link to the Creative Commons licence, and indicate if changes were made. The images or other third party material in this article are included in the article's Creative Commons licence, unless indicated otherwise in a credit line to the material. If material is not included in the article's Creative Commons licence and your intended use is not permitted by statutory regulation or exceeds the permitted use, you will need to obtain permission directly from the copyright holder. To view a copy of this licence, visit http://creativecommons.org/licenses/by/4.0/. 
in [4], and asymptotically linear in [5]. Some special types of solution to (1.1) have also been considered in this direction. For instance, the existence of bound state solutions was considered in [6]. Bump type nodal solutions and sigh-changing solutions were studied in [7-10]. For the case of $V(x)$ and $f(x, u)$ being periodic, the authors in [11,12] obtained the existence of nontrivial solutions for (1.1).

In this paper, we focus on the existence of infinitely many nontrivial solutions of (1.1) by applying variational methods. In variational theory, lots of tools can be used to obtain multiple solutions for equations which have variational structures, such as D.C. Clark's theory for functionals bounded below $[13,14]$ and mountain pass theorem for even functionals [15]. Among these tools, fountain theorem is an important one. Fountain theorem and its dual form were established by Bartsch in [16] and by Bartsch and Willem in [17] (see also [18]) respectively. They are powerful tools to find multiple critical points for variational functionals. Zou in [19] (see also in [20]) established variant fountain theorems without (P.S.) condition, which is a crucial condition for fountain theorem and its dual form. By using the variant fountain theorems given in [19, 20], Zhang and Xu obtained the existence of infinitely many nontrivial solutions of (1.1) in [21], where they did not suppose the common used condition that $\frac{f(x, u)}{u} \rightarrow 0$ as $u \rightarrow 0$, which was used in [19]. Then in [22], Tang weakened some conditions in [21] and gave some more general superquadratic conditions near infinity for the primitive of $f(x, u)$, and finally showed that (1.1) possesses infinitely many nontrivial solutions by applying a result in [15]. Motivated by the work in $[21,22]$, we try to establish the existence of infinitely many high energy solutions of (1.1) under a more general super-quadratic condition than those in [21, 22]. So-called infinitely high energy solutions for (1.1) are solution sequences $\left\{u_{n}\right\}$ of (1.1) such that the corresponding energy denoted by the energy functional of (1.1) goes to infinity as $n \rightarrow \infty$.

To state our result of the present paper, we make the following assumptions on the potential $V$ and nonlinearity $f$ :

$\left(V_{1}\right) V(x) \in C\left(\mathbb{R}^{N}, \mathbb{R}\right)$ is bounded below.

$\left(V_{2}\right)$ For every $M>0$,

$$
\operatorname{meas}\left(\left\{x \in \mathbb{R}^{N}: V(x) \leq M\right\}\right)<\infty
$$

where meas $(\cdot)$ denotes the Lebesgue measure in $\mathbb{R}^{N}$.

$\left(S_{1}\right) f(x, u) \in C\left(\mathbb{R}^{N} \times \mathbb{R}\right)$ and $f(x,-u)=-f(x, u), \forall(x, u) \in \mathbb{R}^{N} \times \mathbb{R}$.

$\left(S_{2}\right)$ There exist constants $c_{1}, c_{2}>0, p, q \in(1,2)$ and $2<p<2^{*}$ such that

$$
|f(x, u)| \leq c_{1}|u|+c_{2}|u|^{p-1}, \quad \forall(x, u) \in \mathbb{R}^{N} \times \mathbb{R} .
$$

$\left(S_{3}\right) \lim _{|u| \rightarrow \infty} \frac{f(x, u)}{u}=\infty$.

$\left(S_{4}\right)$ There exist a constant $\mu>2$ and a function $v(x)>0$ with $|v|_{L^{\infty}}<\infty$ such that

$$
\mu F(x, u) \leq u f(x, u)+v(x) u^{2}, \quad \forall(x, u) \in \mathbb{R}^{N} \times \mathbb{R} .
$$

Then the main result of this paper can be read as follows.

Theorem 1.1 Assume that $V$ and $f$ satisfy $\left(V_{1}\right),\left(V_{2}\right)$ and $\left(S_{1}\right)-\left(S_{4}\right)$. Then problem (1.1) possesses infinitely many high energy solutions. 
Remark 1.2 Comparing with the multiple existent results on nontrivial solutions of (1.1) in [21, 22], Theorem 1.1 obtains infinitely many high energy solutions for (1.1) not only for infinitely many nontrivial solutions. Moreover, we do not make more assumptions on primitive $F(x, u)$ of $f(x, u)$. Specifically, Zhang and Xu in [21] assumed that $F(x, u) \geq 0$ for $\forall(x, u) \in \mathbb{R}^{N} \times \mathbb{R}$ and $\lim _{|u| \rightarrow \infty} \frac{F(x, u)}{|u|^{2}}=\infty$. In [22], Tang improved the conditions in [21] and employed a condition that $F(x, u) \geq 0$ for $|u| \geq r_{0} \geq 0$ and $\lim _{|u| \rightarrow \infty} \frac{F(x, u)}{|u|^{2}}=\infty$. However, we only assume the condition in this paper that

$$
\lim _{|u| \rightarrow \infty} \frac{f(x, u)}{u}=\infty
$$

It is worth mentioning that the condition $\lim _{|u| \rightarrow \infty} \frac{F(x, u)}{|u|^{2}}=\infty$ was also used in [12]. It was also supposed there that $\frac{f(x, t)}{|t|}$ was strictly increasing and $f(x, t)=o(|t|)$ as $|t| \rightarrow 0$ uniformly for $x \in \mathbb{R}^{N}$.

Remark 1.3 To obtain the boundedness of (PS) sequence, Zhang and Xu in [21] introduced that there exists a constant $\vartheta \geq 1$ such that

$$
\vartheta \tilde{F}(x, u) \geq \tilde{F}(x, s u), \quad \forall(x, u) \in \mathbb{R}^{N} \times \mathbb{R}, s \in[0,1]
$$

where $\tilde{F}(x, u)=u f(x, u)-2 F(x, u)$. Then Tang in [22] improved the super-quadratic conditions in [21] and supposed that there exist $c_{0}>0$ and $\kappa>\max \left\{1, \frac{N}{2}\right\}$ such that

$$
|F(x, u)|^{\kappa} \leq c_{0}|u|^{2 \kappa} \mathcal{F}(x, u), \quad \forall(x, u) \in \mathbb{R}^{N} \times \mathbb{R},|u| \geq r_{0},
$$

where $\mathcal{F}(x, u)=\frac{1}{2} u f(x, u)-F(x, u) \geq 0$.

In [22], Tang also imposed another super-quadratic condition that there exist two constants $\mu>2$ and $\rho>0$ such that

$$
\mu F(x, u) \leq u f(x, u)+\rho u^{2}, \quad \forall(x, u) \in \mathbb{R}^{N} \times \mathbb{R} .
$$

Condition (1.5) can be weakened. Thus in this paper we assume condition $\left(S_{4}\right)$ where $v>0$ is a function instead of $\rho>0$ being a constant.

Remark 1.4 It is not difficult to verify that the functions

$$
\begin{aligned}
& f(x, u)=a(x) u \ln (1+|u|), \\
& f(x, u)=a(x)\left(u^{\frac{3}{2}}+u^{3} \cos u\right)
\end{aligned}
$$

satisfy $\left(S_{1}\right)-\left(S_{4}\right)$, where $a(x)$ is a continuous bounded function with positive lower bound.

Remark 1.5 By $\left(V_{1}\right), V(x)$ is bounded below. Thus there exists a positive constant $\bar{V}$ such that $\tilde{V}(x)=V(x)+\bar{V} \geq V_{0}>0$. Let $\tilde{f}(x, u)=f(x, u)+\tilde{V} u$ for all $(x, u) \in \mathbb{R}^{N} \times \mathbb{R}$. Consider the following system:

$$
\left\{\begin{array}{l}
-\Delta u+\tilde{V}(x) u=\tilde{f}(x, u), \quad x \in \mathbb{R}^{N}, \\
u \in H^{1}\left(\mathbb{R}^{N}\right) .
\end{array}\right.
$$


It is easy to check that (1.8) is equivalent to (1.1). Therefore, in what follows we always assume that $V(x)$ has a positive lower bound.

\section{Proof of Theorem 1.1}

We work in the Hilbert space $E=\left\{u \in H^{1}\left(\mathbb{R}^{N}\right): \int_{\mathbb{R}^{N}}\left(|\nabla u|^{2}+V(x) u^{2}\right) d x<+\infty\right\}$ equipped with inner product $\langle u, v\rangle=\int_{\mathbb{R}^{N}}(\nabla u \nabla v+V(x) u v) d x$. The associated norm is $\|u\|=$ $\left(\int_{\mathbb{R}^{N}}\left(|\nabla u|^{2}+V(x) u^{2}\right) d x\right)^{\frac{1}{2}}$, which is equivalent to the standard norm in $H^{1}\left(\mathbb{R}^{N}\right)$ by $\left(V_{1}\right)$ and Remark (1.5). We will find solutions of (1.1) in $E$. In what follows, we use $\|\cdot\|$ and $\|\cdot\|_{p}$ to denote the norms in $E$ and $L^{p}\left(\mathbb{R}^{N}\right)$, respectively.

For $\lambda \in[1,2]$, we define a functional on $E$

$$
\begin{aligned}
I_{\lambda}(u) & =\frac{1}{2} \int_{\mathbb{R}^{N}}\left(|\nabla u|^{2}+V(x) u^{2}\right) d x-\lambda \int_{\mathbb{R}^{N}} F(x, u) d x \\
& =\frac{1}{2}\|u\|^{2}-\lambda \int_{\mathbb{R}^{N}} F(x, u) d x \\
& =A(u)-\lambda B(u) .
\end{aligned}
$$

Since $2<p<2^{*}, E$ can be imbedded continuously into $L^{p}\left(\mathbb{R}^{N}\right)$. By conditions $\left(S_{1}\right)$ and $\left(S_{2}\right)$, $I_{\lambda}(u)$ is well defined. By [23], $B(u) \in C^{1}(E, \mathbb{R})$ and $B^{\prime}(u): E \rightarrow E^{*}$ is compact, where $E^{*}$ is the dual space of $E$. Thus one has $I_{\lambda}(u) \in C^{1}(E, \mathbb{R})$, and for $u, v \in E$,

$$
I_{\lambda}^{\prime}(u) v=\langle u, v\rangle-\lambda B^{\prime}(u) v=\langle u, v\rangle-\lambda \int_{\mathbb{R}^{N}} f(x, u) v d x
$$

By (2.2), solutions of (1.1) correspond to critical points of $I_{\lambda}$ with $\lambda=1$.

Let $\left\{e_{j}: j \in \mathbb{N}\right\}$ be an orthogonal basis of $E . \forall j \in \mathbb{N}$, write $X_{j}=\operatorname{span}\left\{e_{j}\right\}$, then $E=\overline{\bigoplus_{j \in N} X_{j}}$. Let $Y_{k}=\bigoplus_{j=1}^{k} X_{j}, Z_{k}=\overline{\bigoplus_{j=k}^{\infty} X_{j}}$. Notice that $\operatorname{dim} Y_{k}<\infty$. Now we rewrite Theorem 2.1 of [19], since the existence of infinitely many nontrivial solutions of (1.1) is based on it.

Theorem 2.1 Suppose that the functional $I_{\lambda}$ satisfies:

$\left(F_{1}\right) \forall(\lambda, u) \in[1,2] \times E, I_{\lambda}(-u)=I_{\lambda}(u), I_{\lambda}$ maps a bounded set to a bounded set.

$\left(F_{2}\right) \forall u \in E, B(u) \geq 0$, and $\|u\| \rightarrow \infty, A(u) \rightarrow \infty$ or $B(u) \rightarrow \infty$.

$\left(F_{3}\right)$ There exist $r_{k}>\rho_{k}>0$ such that, for all $\lambda \in[1,2]$,

$$
\alpha_{k}(\lambda)=\inf _{u \in Z_{k},\|u\|=\rho_{k}} I_{\lambda}(u)>\beta_{k}(\lambda)=\max _{u \in Y_{k},\|u\|=r_{k}} I_{\lambda}(u) .
$$

Then $\forall \lambda \in[1,2]$, one has

$$
\alpha_{k}(\lambda) \leq \xi_{k}(\lambda)=\inf _{\gamma \in \Gamma_{k}} \max _{u \in B_{k}} I_{\lambda}(\gamma(u))
$$

where $B_{k}=\left\{u \in Y_{k}:\|u\| \leq r_{k}\right\}, \Gamma_{k}=\left\{\gamma \in C\left(B_{k}, E\right): \gamma\right.$ is odd, $\left.\left.\gamma\right|_{\partial B_{k}}=i d\right\}$. For $\lambda \in[1,2]$, there exists a sequence $\left\{u_{m}^{k}(\lambda)\right\}_{m=1}^{\infty}$ such that

$$
\sup _{m}\left\|u_{m}^{k}(\lambda)\right\|<\infty,
$$

and as $m \rightarrow \infty$,

$$
I_{\lambda}^{\prime}\left(u_{m}^{k}(\lambda)\right) \rightarrow 0, \quad I_{\lambda}\left(u_{m}^{k}(\lambda)\right) \rightarrow \xi_{k}(\lambda) .
$$


Now we give several useful lemmas. The first lemma on compact imbedding is well known and its proof can be found in [15].

Lemma 2.2 By condition $\left(V_{2}\right)$ and $2 \leq p<2^{*}$, the imbedding $E \hookrightarrow L^{p}\left(R^{N}\right)$ is compact.

Lemma 2.3 Assume that $\left(V_{1}\right),\left(V_{2}\right)$, and $\left(S_{1}\right)$ hold, then there exist a number $k_{1} \in \mathbb{Z}^{+}$and a sequence $\left\{\rho_{k}\right\}$ such that, as $\rho_{k} \rightarrow \infty(k \rightarrow \infty)$, it has

$$
\alpha_{k}(\lambda)=\inf _{u \in Z_{k},\|u\|=\rho_{k}} I_{\lambda}(u)>0, \quad \forall k \geq k_{1}
$$

where $\alpha_{k}(\lambda) \geq \frac{\rho_{k}^{2}}{4}, Z_{k}=\overline{\bigoplus_{j=k}^{\infty} X_{j}}=\overline{\operatorname{span}\left\{e_{k}, \ldots\right\}}$

Proof Observe that by conditions $\left(V_{1}\right),\left(V_{2}\right)$ and Lemma 3.8 of [18], it holds

$$
l_{k}(q)=\sup _{u \in Z_{k},\|u\|=1}\|u\|_{q} \rightarrow 0, \quad k \rightarrow \infty
$$

By condition $\left(S_{2}\right)$, there exists a constant $c_{3}, c_{4}>0$ such that, for $\forall(x, u) \in \mathbb{R}^{N}$,

$$
|F(x, u)| \leq c_{3}\left(|u|^{2}+|u|^{p}\right)
$$

(2.5) and (2.1) imply that, for $\forall(\lambda, u) \in[1,2] \times \mathbb{R}$,

$$
\begin{aligned}
I_{\lambda}(u) & =\frac{1}{2}\|u\|^{2}-\lambda \int_{\mathbb{R}^{N}} F(x, u) d x \\
& \geq \frac{1}{2}\|u\|^{2}-\lambda \int_{\mathbb{R}^{N}} c_{3}|u|^{2}+c_{4}|u|^{p} d x \\
& \geq \frac{1}{2}\|u\|^{2}-2\left(c_{3}\|u\|_{2}^{2}+c_{4}\|u\|_{p}^{p}\right) \\
& =\frac{1}{2}\|u\|^{2}-2\left(c_{3} l_{2}^{2}(k)\|u\|^{2}+c_{4} l_{p}^{p}(k)\|u\|^{p}\right) .
\end{aligned}
$$

By (2.4), there exists $k_{1}$ such that, for any $k>k_{1}$, one has $l_{2}(k)<\sqrt{\frac{1}{16 c_{3}}}$. Now we take $\rho_{k}=\frac{1}{2}\left(\frac{1}{16 c_{4} l_{p}^{p}(k)}\right)^{\frac{1}{p-2}}$. Then a direct computation implies $I_{\lambda}(u)>\frac{\rho_{k}^{2}}{4}$ for $u \in Z_{k}$ with $\|u\|=\rho_{k}$. Moreover, by $l_{p}^{p}(k) \rightarrow 0$, it holds $\rho_{k} \rightarrow \infty$ as $k \rightarrow \infty$.

Next we prove the following lemma.

Lemma 2.4 Suppose that $\left(V_{1}\right),\left(V_{2}\right),\left(S_{1}\right)-\left(S_{3}\right)$ hold. Then, for $k_{1} \in \mathbb{Z}^{+}$and the sequence $\left\{\rho_{k}\right\}$ in Lemma 2.3, there exists $r_{k}>0$ such that, for $r_{k}>\rho_{k}$,

$$
\beta_{k}(\lambda)=\max _{u \in Y_{k},\|u\|=r_{k}} I_{\lambda}(u)<0, \quad \forall k \geq k_{1}
$$

where $k \in \mathbb{N}, Y_{k}=\bigoplus_{j=1}^{k} X_{j}=\operatorname{span}\left\{e_{1}, \ldots, e_{k}\right\}$ 
Proof By the proof of Lemma 2.6 in [21], for any finite dimensional subspace $\tilde{E} \subset E$, there exists a constant $\epsilon>0$ such that

$$
\operatorname{meas}\left\{x \in \mathbb{R}^{N}:|u(x)| \geq \epsilon\|u\|\right\} \geq \epsilon, \quad \forall u \in \tilde{E}, u \neq 0 .
$$

Therefore, by the fact that the dimension of $Y_{k}$ is finite, there exists a constant $\epsilon_{k}>0$ such that

$$
\operatorname{meas}\left(\Omega_{u}^{k}\right) \geq \epsilon_{k}, \quad \forall u \in Y_{k}, u \neq 0,
$$

where $\Omega_{u}^{k}=\left\{x \in \mathbb{R}^{N}:|u(x)| \geq \epsilon_{k}\|u\|\right\}$ for all $k \in \mathbb{N}$. By $\left(S_{3}\right)$, for each $k \in \mathbb{N}$ and $m_{1}>\frac{1}{2}$, there exists a constant $\zeta_{k}>0$ such that

$$
f(x, u) \geq \frac{2 m_{1} u}{\epsilon_{k}^{3}}, \quad \forall x \in \mathbb{R}^{N},|u| \geq \zeta_{k}
$$

which yields that

$$
F(x, u) \geq \frac{m_{1} u^{2}}{\epsilon_{k}^{3}}, \quad \forall x \in \mathbb{R}^{N},|u| \geq \zeta_{k}
$$

Then, for any $k \in \mathbb{N}$ and $\lambda \in[1,2]$, one has

$$
\begin{aligned}
I_{\lambda}(u) & =\frac{1}{2}\|u\|^{2}-\lambda \int_{\mathbb{R}^{N}} F(x, u) d x \\
& \leq \frac{1}{2}\|u\|^{2}-\lambda \int_{\Omega_{u}^{k}} \frac{m_{1}|u|^{2}}{\epsilon_{k}^{3}} d x \\
& \leq \frac{1}{2}\|u\|^{2}-\epsilon_{k}^{2}\|u\|^{2} m_{1} \frac{\operatorname{meas}\left(\Omega_{u}^{k}\right)}{\epsilon_{k}^{3}} \\
& \leq \frac{1}{2}\|u\|^{2}-m_{1}\|u\|^{2}=-\left(m_{1}-\frac{1}{2}\right)\|u\|^{2},
\end{aligned}
$$

where $u \in Y_{k}$ with $\|u\| \geq \frac{\zeta_{k}}{\epsilon_{k}}$. Choose $r_{k}>\max \left\{\zeta_{k}, \frac{\zeta_{k}}{\epsilon_{k}}\right\}$ for any $k \geq k_{1}$. Then, by $m_{1}>\frac{1}{2}$, it holds

$$
\beta_{k}(\lambda)=\max _{u \in Y_{k},\|u\|=r_{k}} I_{\lambda}(u) \leq-\left(m_{1}-\frac{1}{2}\right) r_{k}^{2}<0 .
$$

Now we are ready to give the proof of Theorem 1.1.

Proof of Theorem 1.1 First of all, by (2.1), (2.2), and (2.5), $I_{\lambda}$ maps bounded sets to bounded sets uniformly for $\lambda \in[1,2]$. By $\left(S_{1}\right)$, we have $I_{\lambda}(-u)=I_{\lambda}(u)$ for $(\lambda, u) \in[1,2] \times E$, which implies that condition $\left(F_{1}\right)$ of Theorem 2.1 holds. It is easy to see that $\left(F_{2}\right)$ holds by $\left(S_{3}\right)$ and (2.1). Condition $\left(F_{3}\right)$ is also true due to Lemma 2.3 and Lemma 2.4. Thus, by Theorem 2.1, there exists a sequence $\left\{u_{m}^{k}(\lambda)\right\}_{m=1}^{\infty}$ for every $k \geq k_{1}$ and $\lambda \in[1,2]$ such that

$$
\sup _{m}\left\|u_{m}^{k}(\lambda)\right\|<\infty, \quad I_{\lambda}^{\prime}\left(u_{m}^{k}(\lambda)\right) \rightarrow 0, \quad I_{\lambda}\left(u_{m}^{k}(\lambda)\right) \rightarrow \xi_{k}(\lambda), \quad \text { a.e. on } \mathbb{R}^{N}
$$


as $m \rightarrow \infty$, where $\xi_{k}(\lambda)=\inf _{\gamma \in \Gamma_{k}} \max _{u \in B_{k}} I_{\lambda}(\gamma(u)), B_{k}=\left\{u \in Y_{k}:\|u\| \leq r_{k}\right\}, \Gamma_{k}=\{\gamma \in$ $C\left(B_{k}, E\right): \gamma$ is odd, $\left.\left.\gamma\right|_{\partial B_{k}}=i d\right\}$. By (2.14), one can choose a sequence $\left\{\lambda_{n}\right\}$ satisfying $\lambda_{n} \rightarrow 1$ for each $k \geq k_{1}$. Moreover, we obtain a sequence $\left\{u_{m}^{k}\left(\lambda_{n}\right)\right\}_{m=1}^{\infty}$ which satisfies as $m \rightarrow \infty$

$$
\sup _{m}\left\|u_{m}^{k}\left(\lambda_{n}\right)\right\|<\infty, \quad I_{\lambda_{n}}^{\prime}\left(u_{m}^{k}\left(\lambda_{n}\right)\right) \rightarrow 0
$$

Note that $\left\{u_{m}^{k}\left(\lambda_{n}\right)\right\}_{m=1}^{\infty}$ is bounded for $m$. So it follows from the compactness of $B^{\prime}$ that $\left\{u_{m}^{k}\left(\lambda_{n}\right)\right\}_{m=1}^{\infty}$ has a strongly convergent subsequence. We denote it still by $\left\{u_{m}^{k}\left(\lambda_{n}\right)\right\}$. We can suppose that

$$
\lim _{m \rightarrow \infty} u_{m}^{k}\left(\lambda_{n}\right)=u_{n}^{k} \in E, \quad \forall n \in \mathbb{N}, k \geq k_{1}
$$

Then by (2.15) it holds

$$
I_{\lambda_{n}}^{\prime}\left(u_{n}^{k}\right)=0, \quad I_{\lambda_{n}}\left(u_{n}^{k}\right) \geq \bar{\rho}_{k}=\frac{\rho_{k}^{2}}{4}
$$

Now we claim that $\left\{u_{n}^{k}\right\}_{n=1}^{\infty}$ is bounded. We prove the claim by contradiction. For convenience, we write $u_{n}^{k}=u_{n}$ for $n \in \mathbb{N}$. Suppose that $\left\|u_{n}\right\| \rightarrow \infty$. Write $v_{n}=\frac{u_{n}}{\left\|u_{n}\right\|}$. Then it is obvious that $\left\|v_{n}\right\|=1$ and $\left\|v_{n}\right\|_{p} \leq \tau\left\|v_{n}\right\|=\tau$ for $2 \leq p<2^{*}$, where $\tau$ is a positive constant by Lemma 2.2. Then by (2.1), (2.2), (2.14), (2.15), and assumption $\left(S_{4}\right)$, one has, for $n$ being large,

$$
\begin{aligned}
c+1 & \geq I_{\lambda}\left(u_{n}\right)-\frac{1}{\mu} I_{\lambda}^{\prime}\left(u_{n}\right) u_{n} \\
& =\frac{\mu-2}{\mu}\left\|u_{n}\right\|^{2}+\int_{\mathbb{R}^{N}}\left(\frac{1}{\mu} f\left(x, u_{n}\right) u_{n}-F\left(x, u_{n}\right)\right) d x \\
& \geq \frac{\mu-2}{\mu}\left\|u_{n}\right\|^{2}-\frac{|v|_{L^{\infty}}}{\mu}\left\|u_{n}\right\|_{2}^{2},
\end{aligned}
$$

which means

$$
1 \leq \frac{2|v|_{L^{\infty}}}{\mu-2} \limsup _{n \rightarrow \infty}\left\|v_{n}\right\|_{2}^{2}
$$

Passing to a subsequence, we can assume that

$$
\begin{aligned}
& v_{n} \rightarrow v_{0} \text { in } E ; \\
& v_{n} \rightarrow v_{0} \quad \text { in } L^{p}\left(\mathbb{R}^{N}\right) \text { for } 2 \leq p<2^{*} ; \\
& v_{n}(x) \rightarrow v_{0}(x) \quad \text { a.e. on } \mathbb{R}^{N} .
\end{aligned}
$$

Therefore it follows from (2.19) and (2.20) that $v_{0} \neq 0$, which implies that meas $(\Sigma)>0$, where $\Sigma=\left\{x \in \mathbb{R}^{N}: v_{0}(x) \neq 0\right\}$. Since $\left\|u_{n}\right\| \rightarrow \infty$ as $n \rightarrow \infty$, we get $\left|u_{n}\right| \rightarrow \infty$ as $n \rightarrow \infty$ on the set $\Sigma$. Then, by $\left(S_{3}\right)$ and (2.17), one has

$$
o(1)=I_{\lambda_{n}}^{\prime}\left(u_{n}\right) u_{n}=\left\|u_{n}\right\|^{2}-\lambda_{n} \int_{\mathbb{R}^{N}} f\left(x, u_{n}\right) u_{n} d x
$$


which implies that

$$
\begin{aligned}
o(1)+1 & =\lambda_{n} \int_{\mathbb{R}^{N}} \frac{f\left(x, u_{n}\right) u_{n}}{\left\|u_{n}\right\|^{2}} d x \\
& \geq \lambda_{n} \int_{\Sigma} \frac{f\left(x, u_{n}\right) u_{n}}{\left\|u_{n}\right\|^{2}} d x \\
& =\lambda_{n} \int_{\Sigma} \frac{f\left(x, u_{n}\right) u_{n}}{\left|u_{n}\right|^{2}}\left|v_{n}\right|^{2} d x \\
& =\lambda_{n} \int_{\Sigma} \frac{f\left(x, u_{n}\right)}{u_{n}}\left|v_{n}\right|^{2} d x \rightarrow \infty,
\end{aligned}
$$

as $n \rightarrow \infty$. This contradiction concludes the boundedness of $\left\{u_{n}\right\}_{n=1}^{\infty}$. Thus we get the claim. By the claim and (2.17), it can be shown by a standard way that there exists a strongly convergent subsequence of $\left\{u_{n}^{k}\right\}$. Suppose that $u_{n}^{k} \rightarrow u_{0}^{k}$ as $n \rightarrow \infty$. Then by (2.17) one has $I_{1}^{\prime}\left(u_{0}^{k}\right)=0$, i.e., $u_{0}^{k}$ is a critical point of $I_{1}(u)$. By (2.17) again, one has $I_{1}\left(u_{0}^{k}\right) \geq \bar{\rho}_{k} \rightarrow$ $\infty$ as $k \rightarrow \infty$. This fact means that equation (1.1) possesses infinitely many high energy solutions. The proof of Theorem 1.1 is complete.

\section{Acknowledgements}

The authors would like to express sincere thanks to the anonymous referees for their careful reading of the manuscript and valuable comments and suggestions.

\section{Funding}

The paper is supported by the National Natural Science Foundation of China (No. 11671077)

Availability of data and materials

Not applicable.

\section{Competing interests}

The authors declare that they have no competing interests.

\section{Authors' contributions}

The authors declare that this study was independently finished. All authors read and approved the final manuscript.

\section{Publisher's Note}

Springer Nature remains neutral with regard to jurisdictional claims in published maps and institutional affiliations.

Received: 11 December 2019 Accepted: 6 March 2020 Published online: 14 March 2020

\section{References}

1. Liu, S., Li, S.: Infinitely many solutions for a superlinear elliptic equation. Acta Math. Sinica (Chin. Ser.) 46, 625-630 (2003) (in Chinese)

2. Ding, Y: Infinitely many entire solutions of an elliptic system with symmetry. Topol. Methods Nonlinear Anal. 9, 313-323 (1997)

3. Jeanjean, L.: On the existence of bounded Palais-Smale sequences and application to a Landesman-Lazer type problem set on $\mathbb{R}^{N}$. Proc. R. Soc. Edinb., Sect. A 129, 787-809 (1999)

4. Kristály, A.: Multiple solutions of a sublinear Schrödinger equation. NoDEA Nonlinear Differ. Equ. Appl. 14, 291-301 (2007)

5. Liu, Z., Su, J., Weth, T:: Compactness results for Schrödinger equations with asymptotically linear terms. J. Differ. Equ. $231,501-512(2006)$

6. Liu, Z., Wang, Z., Weth, T.: Multiple solutions of nonlinear Schrödinger equations via flow invariance and Morse theory. Proc. R. Soc. Edinb., Sect. A 136, 945-969 (2006)

7. Liu, Z., Wang, Z.: Multi-bump type nodal solutions having a prescribed number of nodal domains: II. Ann. Inst. Henri Poincaré, Anal. Non Linéaire 22, 609-631 (2005)

8. Bartsch, T., Liu, Z., Weth, T.: Sign changing solutions of superlinear Schrödinger equations. Commun. Partial Differ. Equ. 29, 25-42 (2004)

9. Bartsch, T., Wang, Z., Willem, M.: The Dirichlet problem for superlinear elliptic equations. In: Chipot, M., Quitter, P. (eds.) Handbook of Differential Equations: Stationary Partial Differential Equations, vol. 2, pp. 1-55. Elsevier, Amsterdam (2005)

10. Bartsch, T., Wang, Z:: Sign changing solutions of nonlinear Schrödinger equations. Topol. Methods Nonlinear Anal. 13, 191-198 (1999) 
11. Li, G., Szulkin, A.: An asymptotically periodic Schrödinger equation with indefinite linear part. Commun. Contemp. Math. 4, 763-776 (2002)

12. Tang, X.: Non-Nehari manifold method for asymptotically periodic Schrödinger equations. Sci. China Math. 58, 715-728 (2015)

13. Clark, D.C.: A variant of the Ljusternik-Schnirelman theory. Indiana Univ. Math. J. 22, 65-74 (1972)

14. Heinz, H.P.: Free Ljusternik-Schnirelman theory and the bifurcation diagrams of certain singular nonlinear systems. J. Differ. Equ. 66, 263-300 (1987)

15. Rabinowitz, P.H.: Minimax Methods in Critical Point Theory with Applications to Differential Equations. CBMS Reg. Conf. Ser. Math., vol. 65. Am. Math. Soc., Providence (1986)

16. Bartsch, T: Infinitely many solutions of a symmetric Dirichlet problem. Nonlinear Anal. TMA 20, 1205-1216 (1993)

17. Bartsch, T., Willem, M.: On an elliptic equation with concave and convex nonlinearities. Proc. Am. Math. Soc. 123, 3555-3561 (1995)

18. Willem, M.: Minimax Theorems. Birkhäuser, Boston (1996)

19. Zou, W.: Variant fountain theorems and their applications. Manuscr. Math. 104, 343-358 (2001)

20. Zou, W., Schechter, M.: Critical Point Theory and Its Applications. Springer, Berlin (2006)

21. Zhang, Q., Xu, B.: Multiplicity of solutions for a class of semilinear Schrödinger equations with sign-changing potential. J. Math. Anal. Appl. 377, 834-840 (2011)

22. Tang, $X$ :. Infinitely many solutions for semilinear Schrödinger equations with sign-changing potential and nonlinearity. J. Math. Anal. Appl. 401, 407-415 (2013)

23. Rabinowitz, P.H.: On a class of nonlinear Schrödinger equations. Z. Angew. Math. Phys. 43, 270-291 (1992)

\section{Submit your manuscript to a SpringerOpen ${ }^{\circ}$ journal and benefit from:}

- Convenient online submission

- Rigorous peer review

- Open access: articles freely available online

- High visibility within the field

- Retaining the copyright to your article

Submit your next manuscript at $\gg$ springeropen.com 